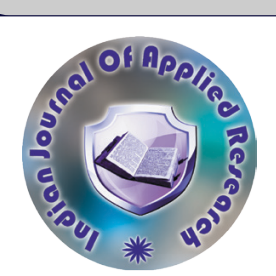

Microbiology

\title{
STUDY OF THREE MALARIA RAPID DETECTION TESTS AND ITS CORRELATION WITH PARASITIC INDEX FOR P.FALCIPARUM AND P.VIVAX
}

Dr. Dipti Gaikwad

\section{Dr. Chaya A. Kumar*}

Dr. Sujata Baveja
Postgraduate Resident, Department Of Microbiology, Lokmanya Tilak Municipal Medical College And General Hospital, Sion, Mumbai.

Associate Professor, Department Of Microbiology, Lokmanya Tilak Municipal

Medical College And General Hospital, Sion, Mumbai. *Corresponding Author

Professor And HOD, Department Of Microbiology, Lokmanya Tilak Municipal

Medical College And General Hospital, Sion, Mumbai.

ABSTRACT) OBJECTIVE: To determine the sensitivity and specificity of three Malaria rapid antigen detection tests(RDTs) .To study the sensitivity of the RDTs in relation to parasitic index. MATERIALS AND METHODS: The study was conducted at a tertiary care hospital. Peripheral smear were prepared and stained.Parasite index was calculated. Three rapid antigen detection tests ; Optimal - IT, Paramax-3 and QDx malaria PAN/PF were tested. RESULTS: Sensitivity for Optimal - IT was highest (98.47\%). QDx malaria PAN/Pf gave highest specificity (97\%). All the three RDTs gave sensitivity of $100 \%$ at a parasitic index of more than 100 parasites per $\mu 1$. Sensitivity of Optimal - IT and QDx malaria PAN/Pf for P falciparum and P vivax was $100 \%$ and $88.88 \%$ respectively at a parasitic index of 51 100 parasites/ $\mu 1$. CONCLUSION: Malaria RDTs are a good diagnostic tool in health care set ups where quick results are desired and expert microscopy is not available.

KEYWORDS : Malaria, Rapid Antigen Detection Tests, Optimal - IT, Malaria Diagnosis, Malaria Parasitic Index

\section{INTRODUCTION}

Malaria has been known since ancient times and has been diagnosed based on patient's signs and symptoms. Today with increasing chloroquine resistance and widespread use of expensive arteminisin combination therapies, an accurate and rapid diagnosis is essential. Clinical features of malaria have been described from Hippocratus to Osler. ${ }^{1}$ In 1891, Romanowky introduced staining methods for these parasites. ${ }^{2,3}$ Today more than a century later, microscopic detection and identification of Plasmodium species in Giemsa stained blood films remains the gold standard for laboratory diagnosis. Methods using advances in technology have been introduced as alternatives to light microscopy. ${ }^{4}$ Becton and Dickenson developed a fluorescent staining technique using a capillary tube called as Quantitative Buffy Coat test in 1991-92. ${ }^{2}$ Polymerase chain reaction (PCR) based methods have been used since early 1900's for the detection of Plasmodium parasites in human patients. ${ }^{5}$ Many non-microscopic, rapid dip stick tests based on the detection of various antigens of malaria parasites were introduced in the early 1990 s. $^{6}$ P.falciparum Histidine Rich Protein II (1987), parasite aldolase ( 1992) and parasite Lactate dehydrogenase (1998) are the target antigens used for such tests.

Several commercial kits are available detecting different malaria antigens like the Histidine rich protein 2 (HRP-2), parasite lactate dehydrogenase $(\mathrm{pLDH})$ and aldolase. These tests can be performed in 15-20 minutes. Reporting the test results of the Rapid detection tests (RDT's) also does not require the expertise as it is for microscopy. There are several Rapid antigen detection tests available. The choice of an appropriate assay would help in accurate and rapid malaria diagnosis.

\section{MATERIALAND METHODS}

The present study was carried out at a tertiary care hospital after approval by the Ethical committee of the parent college.

Two hundred blood samples were obtained from patients of all ages with clinical suspicion of malaria. Patients who came for follow-up visits of an earlier episode of malaria or within 4 weeks post treatment were excluded from the study. Patients with history of treatment with antimalarials were excluded from the study.

From each case $2 \mathrm{ml}$. of blood was collected into an ethylenediamine tetra acetic acid bulb(EDTA) and one thick and thin blood film each was prepared on different slides and stained with Giemsa stain as per standard protocol. A thick smear was considered negative if no parasites were seen in at least 200 fields.? white blood cells (WBCs) and the parasite index was calculated using the following formula.

\section{$\underline{\text { Parasites counted }} \times 8000=$ parasite count per microlitre of blood \\ 200}

Thin films were examined for at least 100 fields in not more than 10 minutes. ${ }^{3}$ Speciation of the parasites was done by examining stained thin films. Three malaria rapid antigen detection kits (RDTs) were used and compared with peripheral smear. The three RDTs were

Optimal - IT (DiaMed, Switzerland), Paramax -3 (Zephyr Biomedicals, India), and QDx MALARIA PAN / Pf (Piramal Healthcare, India).

\section{Optimal - IT (DiaMed, Switzerland)}

DiaMed Optimal - It is an immunochromatogrphic test, using monoclonal antibodies against the metabolic enzyme pLDH of Plasmodium spp. These monoclonal antibodies are classified in two groups; one specific for Plamodium falciparum and the other is a pan specific monoclonal antibody which reacts with all four species of Plasmodium spp. which can occur in human beings: P.falciparum, P.vivax, P.ovale, P.malariae

\section{Paramax -3 (Zephyr Biomedicals, India)}

It is a rapid, qualitative, two site sandwich immunoassay utilizing whole blood for the detection of P.falciparum specific histidine rich protein-2 (Pf. HRP-2), P.vivax specific pLDH and pan malaria specific pLDH.

\section{QDx MALARIA PAN / Pf (Piramal Healthcare, India)}

QDx Malaria PAN / Pf is a qualitative, sandwich immunoassay for the detection and differentiation of P.falciparum specific histidine rich protein-2 (Pf. HRP-2) and pan malaria specific $\mathrm{pLDH}$.

Rapid diagnostic tests were read by the same bacteriologist and confirmed by a second independent reader when needed, allaccording to the manufacturer's instructions.

Statistical analysis: The performance of RDTs was expressed by calculating the sensitivity, specificity, negative predictive value (NPV), and positive predictive value (PPV), for P. vivax and $\mathrm{P}$. falciparum separately, taking microscopy results as the "gold standard". The sensitivity and specificity of the gold standard was taken as $95 \%$. The sample size of the study was 200 . Based on this, standard error was calculated to be 1.54 . The normal range of sensitivity and specificity is $95 \% 1.54 \%$ ie $93.46 \%-96.54 \%$.Data were analyzed in SPSS. Proportions were compared using the Chi-square 


\section{TABLE 4}

\section{RESULTS}

Of the 200 samples processed in the laboratory, 132 had positive blood films. Ninety four of the 132 infecting species were identified as P.vivax, 33 as P.falciparum, and 5 as mixed infections.

To assess the performance of optimal-IT and QDx MALARIA PAN / Pf for diagnosis of P.vivax, cases with mixed infections with P.falciparum were excluded, because the pan-malaria antigen turns up positive due to P.falciparum infection. For performance of P.falciparum detection, mixed infections with P.falciparum were included. One slide which had only gametocytes was not included in further analysis.

\section{TABLE 1}

Based on the above observations OptiMAL-IT had a sensitivity and specificity of $98.47 \%$ and $95.58 \%$ respectively. The Positive predictive value (PPV) and Negative predictive value (NPV) was $97.72 \%$ and $97.01 \%$ respectively.

Sensitivity and specificity obtained for Paramax-3 was $95.41 \%$ and $94.11 \%$ respectively. It had a PPV of $96.89 \%$ and NPV of $91.42 \%$.

The sensitivity and specificity recorded for QDxMALARIA PAN/Pf was $96.94 \%$ and $97.05 \%$ respectively. The PPV and NPV was $98.44 \%$ and $94.28 \%$ respectively.

The confidence interval calculated for the sensitivity and specificity of Giemsa stained peripheral blood smear which was the gold standard was found to be $93.46 \%-96.54 \%$. The sensitivity and specificity obtained for QDxMALARIA PAN/Pf was higher than this confidence interval, thus giving better results than microscopy. The sensitivity for OptiMAL-IT was also higher than the normal range for microscopy. The specificity for OptiMAL-IT and sensitivity and specificity for Paramax-3 is within the confidence interval giving comparable results with microscopy.

\section{TABLE 2}

Sensitivity of OptiMAL-IT for P.falciparum was $97.29 \%$. The specificity recorded was $99.38 \%$. The PPV and NPV obtained was $97.29 \%$ and $99.38 \%$ respectively.

Based on the above observations Paramax-3 had a sensitivity and specificity of $94.59 \%$ and $98.76 \%$ respectively for P.falciparum. The PPV and NPV was $94.59 \%$ and $98.76 \%$ respectively.

QDxMALARIA PAN/Pf recorded a sensitivity and specificity of 97.29 $\%$ and $99.38 \%$ respectively when tested for P.falciparum. The PPV observed was $97.29 \%$ while the NPV was $99.38 \%$.

The sensitivity and specificity for OptiMAL-IT and QDxMALARIA PAN/Pf for P.falciparum was higher than the upper limit of confidence interval (93.46\%- 96.54\%). The specificity for Paramax-3 was also high while its sensitivity was within the range. Thus rapid tests gave either better or comparable results for P.falciparum when compared with microscopy.

\section{TABLE 3}

Sensitivity of OptiMAL-IT for P.vivax was $98.93 \%$. The specificity recorded was $98.00 \%$. The PPV and NPV obtained was $97.89 \%$ and $98.98 \%$ respectively.

Paramax-3 had a sensitivity and specificity of $95.95 \%$ and $98.00 \%$ respectively for P.vivax. The PPV and NPV was $97.93 \%$ and $96.07 \%$ respectively.

QDXMALARIAPAN/Pf recorded a sensitivity and specificity of 96.80 $\%$ and $99.00 \%$ respectively when tested for P.vivax. The PPV observed was $98.91 \%$ while the NPV was $97.05 \%$.

The sensitivity and specificity for OptiMAL-IT and QDxMALARIA PAN/Pf for P.vivax was higher than the upper limit of confidence interval (93.46\%- 96.54\%). The specificity for Paramax-3 was also high while its sensitivity was within the range. Thus OptiMAL-IT and QDxMALARIA PAN/Pf for P.vivax gave better results when compared with microscopy. Paramax-3 also gave comparable results with microscopy.
Percentage sensitivity for rapid tests with relation to parasitic index was lower at an index of $0-50$ parasites / $\mu$ l for P.falciparum cases. Percentage sensitivity for Paramax-3 at parasitic index 50-100 parasites / $\mu \mathrm{l}$ was lower than other rapid tests. Overall percentage sensitivity was higher for OptiMAL-IT and QDxMALARIA PAN/Pf as compared to Paramax-3 for P.falciparum cases.

\section{TABLE 5}

The overall percentage sensitivity with relation to parasitic index was highest for OptiMAL-IT for P.vivax cases. OptiMAL-IT gave a sensitivity of $100 \%$ at a parasitic index of $0-50$ parasites $/ \mu 1$ but one case was found to be negative with a parasitic index of $51-100$ parasites $/ \mu 1$.

\section{DISCUSSION}

The aim of the study was to compare three rapid antigen detection tests with peripheral blood smear for the diagnosis of malaria. The number of cases were limited to only symptomatic indoor patients with clinical suspicion of malaria. Sub-clinical infection or normal controls were not included in the study, hence the number of samples with low parasitaemia ( $<100$ per cumm) and gametocytes only, were few in the study.

It was found that rapid tests yielded comparable results with microscopy.

OptiMAL-IT a pLDH based assay had an overall sensitivity of $98.47 \%$ and a specificity of $95.58 \%$. The positive predictive value (PPV) was $97.72 \%$ and negative predictive value (NPV) was $97.01 \%$. It had a sensitivity of $97.29 \%$ and specificity of $99.38 \%$ for P.falciparum and sensitivity of $98.93 \%$ and specificity of $98 \%$ for P.vivax. QDx MALARIA PAN / Pf gave similar results as OptiMAL-IT. It had an overall sensitivity of $96.94 \%$ and a specificity of $97.05 \%$. The PPV was $98.44 \%$ and NPV of $94.28 \%$. It had a sensitivity of $97.29 \%$ and specificity of $99.38 \%$ for P.falciparum and sensitivity of $96.80 \%$ and specificity of $99 \%$ for P.vivax. The results obtained for Paramax -3 were slightly lower than the above two tests. It showed an overall sensitivity of $95.41 \%$ and a specificity of $94.11 \%$. The PPV was 96.89 $\%$ and NPV of $91.42 \%$. It had a sensitivity of $94.59 \%$ and specificity of $98.76 \%$ for P.falciparum and sensitivity of $95.95 \%$ and specificity of $98 \%$ for P.vivax.

The overall sensitivity and specificity obtained for QDxMALARIA PAN/Pf was higher than the confidence interval calculated for comparing rapid tests with microscopy. Thus it gave better results than microscopy. The sensitivity for OptiMAL-IT was also higher than the normal range for microscopy. The specificity for OptiMAL-IT and sensitivity and specificity for Paramax-3 was within the confidence interval giving comparable results with microscopy.

Sensitivity of rapid tests with relation to parasite index was comparable with microscopy at a parasitic index of $>100$ parasites $/ \mu 1$. However the sensitivity dropped when the parasitic index was below 100 parasites $/ \mu 1$

Several authors who have tested OptiMAL-IT for malarial diagnosis from different countries have given more or less similar results. They have reported a sensitivity and specificity in the range of $80-100 \%$. Palmer et al in Honduras has reported a sensitivity of $94 \%$ and specificity of $100 \%$ for P.vivax and sensitivity of $88 \%$ and specificity of $99 \%$ for P. falciparum. ${ }^{10}$ From hospital of tropical diseases UK, Moody et al while conducting their study in sub-Saharan Africa, Asia and South America have reported a sensitivity of $95.3 \%$ and specificity of $100 \%$ for P. falciparum and sensitivity of $96 \%$ and a specificity of $100 \%$ for Plasmodium vivax. ${ }^{11}$ John et al obtained a sensitivity of $94 \%$ for P. falciparum and $98.2 \%$ for P.vivax in a trial in Southern India. ${ }^{12}$ The sensitivity and specificity of the present study falls in the range given above and is comparable to the various studies. Therefore the results of this study further substantiates that OptiMAL-IT is an effective and sensitive tool in the diagnosis of malaria.

There are no published reports for Paramax-3 and QDx MALARIA PAN / Pf except for the technical support sheet by Malaria Reseach Centre (ICMR) for testing Paramax. ${ }^{13}$ There are several studies on other HRP2 based assays like ICT P.f/P.v, NOW-Malaria-ICT, Paracheck-Pf, ParaSight-F, and Parascreen pan/pf etc. Paramax and Parascreen malaria diagnostic tests manufactured by M/s Zephyr Biomedicals, Goa were tested at Malaria Research Centre (ICMR), 
Goa, India in 2004. Both tests gave a $100 \%$ sensitivity, specificity, NPV, and PPV for both P. falciparum and P. vivax.

The parasitaemia range for $P$. falciparum and $P$. vivax was 400-22,720 and 520-33600 parasites/ $\mu$ l of blood respectively. ${ }^{13}$ In the present study Paramax -3 was $100 \%$ sensitive at a parasitic index of $>100$ parasites $/ \mu 1$.

Among the various HRP2 based tests for malaria, there is a wide range of sensitivity and specificity seen. In the present study the rapid tests showed a sensitivity and specificity at the higher range. The sensitivity and specificity for Paramax -3 was slightly lower than OptiMAL-IT and QDx MALARIA PAN / Pf. However it can identify the infection caused by P.falciparum and P.vivax separately. It can also diagnose mixed infection. The identity of the Plasmodium species helps clinicians to anticipate complications and treat the patients promptly and accurately.

In spite of over hundred published RDT trial reports, comparative assessment is difficult because i) trials do not share common guidelines; ii) clinical and epidemiological characteristics of the study populations, especially parasitemia level vary; iii) reference standards are different; even among those using Giemsa microscopy, reading rules and microscopist's skill vary; and iv) products of different lots may differ in quality or be damaged by extreme temperature or humidity during transportation and storage.

HRP-2 based tests commonly give P.falciparum sensitivity of $>90 \%$ in clinical cases. For pLDH assays, results varied among studies and product lots and variable field stability of the test could not be ruled out. Sensitivity for P.falciparum is excellent $(>95 \%)$ in some studies and poorer $(80 \%)$ in others. Recent studies suggest that the tests were less sensitive for non - P.falciparum than for P.falciparum. Extremely low sensitivity had been reported earlier for both HRP-2 and pLDH tests and batch specific problems were suspected. Overall RDT specificity is commonly above $85 \%$, approaching $100 \%$ when used in some groups of returning non immune travellers. ${ }^{14}$

In the present study OptiMAL-IT missed two cases which were positive on microscopy. Paramax-3 missed 6 cases while QDx MALARIA PAN / Pf missed 4 cases of malaria. The reason for these false negative results could be that rapid tests are not sensitive below a parasitic index of 100 parasite/ul. ${ }^{15}$ This fact is also reflected in the present study, as, all the cases which were missed had a parasitic index below 100 parasite/ul on the peripheral blood smear. Besides, occasional false negative results may be caused by deletion or mutation of the hrp-2 gene. Secondly, It has been suggested that anti HRP-2 antibodies in humans may explain why some tests were negative. Presence of an inhibitor in the patient's blood preventing development of control line has also been noted. ${ }^{14} \mathrm{pLDH}$ is produced by living parasites. It is possible that some patients might have already taken antimalarials and not disclosed it which could also account for negative results of the RDTs. ${ }^{16}$

The rapid tests also gave some false positive results. OptiMAL -IT gave 3 false positive results while Paramax-3 and QDx MALARIA PAN / Pf gave 4 and 2 false positive results respectively. These may be due to a number of factors. Cross reactivity with rheumatoid factor in blood generates a false positive test line, but replacement of IgG with $\mathrm{IgM}$ in recent products reduces this problem. Cross reactivity with heterophile antibodies may also occur. ${ }^{14} \mathrm{HRP}-2$ has been shown to persist and is detectable after the clinical symptoms of malaria have disappeared and the parasites have apparently been cleared from the host. Low level parasitemia seen in areas of endemic infection because of constant exposure to the malarial parasites may also result in positive results with doubtful clinical significance. ${ }^{8}$ Furthermore, in some infections parasites may have been sequestered and would not be detected on peripheral blood smear examination. ${ }^{17}$

It was observed that five cases of mixed malarial infection were seen on microscopy. OptiMAL-IT and QDx MALARIA PAN / Pf reported these cases as P. falciparum due to the configuration of the test. Paramax-3 identified these cases as mixed infections with 100\% sensitivity and specificity. As the management of mixed infections includes P.vivax treatment, the format used in Paramax-3 is advantageous. One case in this study showed only the gametocytes of
P. falciparum in the blood smear and no ring forms were seen. The rapid tests reported it as P. falciparum infestation. Such results can lead to unnecessary medication.

The commerciallyavailable malaria rapid detection tests in kit form are a good alternative to microscopic diagnosis for malaria especially where quick results are required or in health care facilities where there is a lack of manpower and equipments as these tests do not require extensive training or equipments to perform or to interpret the results. Besides, the tests can be performed in 15 to 20 minutes.

\section{CONCLUSIONS}

Peripheral blood smear examination remains the gold standard for diagnosis of malaria while Rapid antigen detection tests are a good alternative but cannot be a substitute. Rapid antigen detection tests have maximum utility in emergency laboratories, intensive care setups and the casualty where quick results are desired. They can also be used for malaria diagnosis where facilities for expert microscopy are not available

Table 1: Results Of Malaria Rapid Detection Tests For Malaria Positive Cases ( $n=199)$

\begin{tabular}{|l|l|l|l|}
\hline \multirow{2}{*}{ Results of RDTs } & \multicolumn{2}{|l|}{ Results of Microscopy } & Total \\
\cline { 2 - 3 } & Positive & Negative & \\
\hline $\begin{array}{l}\text { OptiMAL-IT results (n=199) } \\
\text { Positive }\end{array}$ & 129 & 3 & 132 \\
\hline Negative & 2 & 65 & 67 \\
\hline $\begin{array}{l}\text { Paramax-3 results(n=199) } \\
\text { Positive }\end{array}$ & 125 & 4 & 129 \\
\hline Negative & 6 & 64 & 70 \\
\hline $\begin{array}{l}\text { QDxMALARIAPAN/Pf (n=199) } \\
\text { Positive }\end{array}$ & 127 & 2 & 129 \\
\hline Negative & 4 & 66 & 70 \\
\hline
\end{tabular}

Table 2: Results Of Rapid Tests For P.falciparum

\begin{tabular}{|l|l|l|l|}
\hline \multirow{2}{*}{$\begin{array}{l}\text { Results of RDTs } \\
\text { P.falciparum (n=199) }\end{array}$} & \multicolumn{2}{|l|}{ Results of Microscopy } & Total \\
\cline { 2 - 3 } $\begin{array}{l}\text { OptiMAL-IT P.falciparum } \\
\text { Positive }\end{array}$ & 36 & 1 & \\
\hline Negative & 1 & 161 & 162 \\
\hline $\begin{array}{l}\text { Paramax-3 } \\
\text { Positive }\end{array}$ & 35 & 2 & 37 \\
\hline Negative & 2 & 160 & 162 \\
\hline $\begin{array}{l}\text { QDxMALARIAPAN/Pf } \\
\text { Positive }\end{array}$ & 36 & 1 & 37 \\
\hline Negative & 1 & 161 & 162 \\
\hline
\end{tabular}

Table 3: Results Of Rapid Tests For P.vivax

\begin{tabular}{|l|l|l|l|}
\hline $\begin{array}{l}\text { Results of RDTs } \\
\text { P.vivax (n=194) }\end{array}$ & \multicolumn{2}{|l|}{ Results of Microscopy } & Total \\
\cline { 2 - 4 } $\begin{array}{l}\text { OptiMAL-IT } \\
\text { Positive }\end{array}$ & 93 & 2 & \\
\hline Negative & 1 & 98 & 95 \\
\hline $\begin{array}{l}\text { Paramax-3 } \\
\text { Positive }\end{array}$ & 95 & 2 & 99 \\
\hline Negative & 4 & 98 & 97 \\
\hline $\begin{array}{l}\text { QDxMALARIAPAN/Pf } \\
\text { Positive }\end{array}$ & 91 & 1 & 102 \\
\hline Negative & 3 & 99 & 92 \\
\hline
\end{tabular}

Table 4: Percentage Sensitivity Of Rapid Tests With Relation To Parasitic Index For P.falciparum $(n=32)$ *

\begin{tabular}{|l|l|l|l|l|}
\hline $\begin{array}{l}\text { P.falciparum } \\
(\mathbf{n = 3 2})^{*} \\
\text { Parasites/ } \boldsymbol{\mu l}\end{array}$ & $\begin{array}{l}\text { Microscopy } \\
\text { Results }\end{array}$ & $\begin{array}{l}\text { OptiMAL-IT } \\
\text { positive } \\
\text { (\% Sensitivity) }\end{array}$ & $\begin{array}{l}\text { Paramax-3 } \\
\text { positive } \\
\text { (\% Sensitivity) }\end{array}$ & $\begin{array}{l}\text { QDxMALARIA } \\
\text { PAN/Pf positive } \\
\text { \% Sensitivity) }\end{array}$ \\
\hline $0-50$ & 3 & $2(66.66)$ & $2(66.66)$ & $2(66.66)$ \\
\hline $51-100$ & 3 & $3(100)$ & $2(66.66)$ & $3(100)$ \\
\hline $101-500$ & 3 & $3(100)$ & $3(100)$ & $3(100)$ \\
\hline $501-5,000$ & 7 & $7(100)$ & $7(100)$ & $7(100)$ \\
\hline $5,001-50,000$ & 14 & $14(100)$ & $14(100)$ & $14(100)$ \\
\hline$>50,000$ & 2 & $2(100)$ & $2(100)$ & $2(100)$ \\
\hline Overall & 32 & $31(96.87)$ & $30(93.75)$ & $31(96.87)$ \\
\hline
\end{tabular}


* False positive cases and a case with only gametocytes was not included

Table 5: Percentage Sensitivity Of Rapid Tests With Relation To

Parasitic Index For P.vivax ( $=94$ )*

\begin{tabular}{|l|l|l|l|l|}
\hline $\begin{array}{l}\text { P.vivax } \\
(\mathbf{n = 9 4})^{*} \\
\text { Parasites/} / \boldsymbol{\mu l}\end{array}$ & $\begin{array}{l}\text { Giemsa } \\
\text { positive }\end{array}$ & $\begin{array}{l}\text { OptiMAL-IT } \\
\text { positive } \\
\text { (\% Sensitivity) }\end{array}$ & $\begin{array}{l}\text { Paramax-3 } \\
\text { positive } \\
\text { \%Sensitivity) }\end{array}$ & $\begin{array}{l}\text { QDxMALARIA } \\
\text { PAN/Pf positive } \\
\text { (\% Sensitivity) }\end{array}$ \\
\hline $0-50$ & 9 & $9(100.00)$ & $7(77.77)$ & $7(77.77)$ \\
\hline $51-100$ & 9 & $8(88.88)$ & $7(77.77)$ & $8(88.88)$ \\
\hline $101-500$ & 16 & $16(100)$ & $16(100)$ & $16(100)$ \\
\hline $501-5,000$ & 17 & $17(100)$ & $17(100)$ & $17(100)$ \\
\hline $5,001-50,000$ & 41 & $41(100)$ & $41(100)$ & $41(100)$ \\
\hline$>50,000$ & 2 & $2(100)$ & $2(100)$ & $2(100)$ \\
\hline Overall & 94 & $93(98.93)$ & $90(95.74)$ & $91(96.80)$ \\
\hline
\end{tabular}

* False positive cases were not included.

\section{REFERENCES}

1. Cunha, C. B., Cunha, B. A., (2008), "Brief history of the clinical diagnosis of malaria: from Hippocrates to Osler." Journal of Vector Borne Diseases, 45,194-199.

2. Malaria. http://www.malariasite.com. Accessed on 24. 07.2010.

3. Chatterjee, K, D., "Parasitology: In relation to clinical medicine".12th ed. Chatterjee Medical Publishers; 1980.

4. Tangpukdee, N., Duangdee, C., Wilairatana, P., Krudsood, S.,(2009),“ Malaria Diagnosis: ABrief Review". Korean Journal of Parasitoogy,47(2): 93-102.

5. Coleman,R. E., Sattabangkot, J, Promstaporm, S., Maneechai, N., Tippayachi, B., Kenglucha, A., et al. (2006), "Comparison of PCR and microscopy for the detection of asymptomatic malaria in a Plasmodium falciparum /vivax endemic area in Thailand." asymptomatic malaria in a Plasmodium falciparum /vivax endemic area in Thailand." Malaria Journal; 5,121

6. Bell, D., Wongsrichanalal, C. and Barnwell, J. W. (2006), "Ensuring quality and access for malaria diagnosis: How can it be achieved?" Nature Reviews - Microbiology, Nature Publishing Group, S7-S20.

7. Broek, I. V. D., Hill, O., Gordillo, F., Angarita, B., Hamade, P., Couniha, H., Guthmann, J. P. (2006) " Evaluation of three rapid tests for diagnosis of P.falciparum and P.vivax malaria in Colombia." American Journal of Tropical Medicene and Hygiene, 75(6), $1209-1215$.

8. Moody, A. (2002), "Rapid diagnostic tests for malaria parasites." Clinical Microbiology Reviews, 15(1), 66-78.

9. Murry, C. K., Gasser, R. A., Magill, A. J., Miller, R. C.(2008) "Update on rapid diagnostic testing for malaria." Clinical Microbiology Reviews, 21(1),97-110.

10. Palmer, C. J., Lindo, J. F., Klaskala, W. I., Quesada, J. A., Kaminsky, R., Baum, M. K., Ager, A. L. (1998) "'Evaluation of the OptiMAL test for rapid diagnosis of Plasmodium vivax and Plasmodium falciparum malaria." Journal of Clinical Microbiology, 36(1),203-206.

11. Moody, A., Hunt-Cooke, A., Gabbet, E., Chiodini, P.,( 2000) "Performance of the OptiMAL malaria antigen capture dipstick for malaria diagnosis and treatment monitoring at the hospital and tropical diseases, London." British Journla of monitoring at the hospital a
Haematology,109(4),891-894.

12. John, S. M., Sudarsanam, A., Sitaram, U., Moody, A. H.(1996) Evaluation of OptiMAL, a dipstick test for the diagnosis of malaria. Annals of Tropical Medicine and Parasitology, 92(5), 621-2.

13. Evaluation of parascreen and paramax malaria diagnostic tests manufactured by $\mathrm{M} / \mathrm{s}$ Zephyr Biomedicals, Verna, Goa.

14. Wongsrichanalai, C., Barcus, M. J., Muth ,S., Sutamihardja, A. and Wernsdorfer, W, H. (2007) "A review of malaria diagnostic tools: Microscopy and rapid diagnostic test." American Journal of Tropical Medicine and Hygiene 77(6), 119-127.

15. Congpuong, K., Bualombai, P., Jitchamroen, S., Konchom, S.,(2001) "Comparison of the OptiMAL rapid test with routine microscopic examination of Giems-stained thick blood film for diagnosis of malaria." Journa of the medical Association of Thailand blood film for diagnosis of malaria." Journa of the medical Association of Thailand
$84(3), 357-63$ ,84(3), 357-63.

16. Khan, S. A., Anwar ,M., Hussain, S., Qureshi, H., Ahmed, M. and Afzal, S. (2004) "Comparison of OptiMAL malarial test with light microscopy for the diagnosis of malaria." Journal of the Pakistan Medical Association , 54(8), 404-407.

17. Mendiratta, D. K., Bhutada, K., Narang, R., Narang, P. (2006) "Evaluation of different methods for diagnosis of P.falciparum malaria". Indian Journal of Medical Microbiology ,24(1), 49-51. 